Proyecciones

Vol. 26, $\mathrm{N}^{o}$ 3, pp. 259-267, December 2007.

Universidad Católica del Norte

Antofagasta - Chile

\title{
REGULARITY AND AMENABILITY OF THE SECOND DUAL OF WEIGHTED GROUP ALGEBRAS
}

\author{
A. REJALI \\ ISFAHAN UNIVERSITY, IRÁN \\ and \\ H. R. E. VISHKI \\ FERDOWSI UNIVERSITY, IRÁN \\ Received : April 200\%. Accepted : October 2007
}

\begin{abstract}
For a wide variety of Banach algebras $A$ (containing the group algebras $L^{1}(G), M(G)$ and $A(G)$ ) the Arens regularity of $A^{* *}$ is equivalent to that $A$, and the amenability of $A^{* *}$ is equivalent to the amenability and regularity of $A$. In this paper, among other things, we show that this variety contains the weighted group algebras $L^{1}(G, w)$ and $M(G, w)$.
\end{abstract}

Keywords : Arens product, Weighted group algebra, Amenability

$\operatorname{MR(2000)}$ Subject Classification : 46H25, 43A10 


\section{Introduction}

Over fifty years ago, Arens in his elaborate work [A], pointed out that, for every Banach algebra $A$, there exist two (Arens) products $\circ$ and $\diamond$ on the second dual $A^{* *}$, extending the product of $A$. If these two products coincide on $A^{* *}$, then $A$ is said to be (Arens) regular. For further details on the properties of Arens products see the survey article [D-H]. It is readily verified that the regularity of $A^{* *}$ (equipped with either $\circ$ or $\diamond$ ) implies that of $A$; therefore $\left(A^{* *}, \circ\right)$ is regular if and only if $\left(A^{* *}, \diamond\right)$ is regular. However it has been shown in [Y3] that there exists a regular Banach algebra whose second dual is not regular; for a more simple example of such a Banach algebra see $[\mathrm{P}]$. Every $C^{*}$-algebra is regular $[\mathrm{S}]$, and its second dual is a von Neumann algebra, and so is regular. As a consequence of Young's result [Y1], (which asserts $L^{1}(G)$ is regular if and only if $G$ is finite) the regularity of $L^{1}(G)^{* *}$ is equivalent to the regularity of $L^{1}(G)$. For a commutative, semisimple, completely continuous and weakly sequentially complete Banach algebra $A$ whose dual $A^{*}$ is a von Neumann algebra (for instance, for the Fourier algebra $A(G)$ ), it has been shown in [U2] that the regularity of $A^{* *}$ is equivalent to that of $A$.

A Banach algebra $A$ is said to be amenable (resp. weakly amenable) if every continuous derivation $D: A \rightarrow X^{*}$ (resp. $D: A \rightarrow A^{*}$ ) is inner for every Banach $A$-module $X$. It has been shown in [Go] (see also [G-L$\mathrm{W}])$ that, if either $\left(A^{* *}, \circ\right)$ or $\left(A^{* *}, \diamond\right)$ is amenable then so is $A$. However, for an infinite amenable group $G, L^{1}(G)$ is amenable, but $L^{1}(G)^{* *}$ is not; indeed in [G-L-W] they showed that $L^{1}(G)^{* *}$ is amenable if and only if $G$ is finite. For the Fourier algebra $A(G)$ it is known that, $A(G)^{* *}$ is amenable if and only if $G$ is finite, see [Gra]. Although, one can use the earlier result of Forrest and Runde [F-R], to give a simple proof for the latter fact; (indeed, if $A(G)^{* *}$ is amenable then so is $A(G)$, and the main result of [F-R] implies that, $G$ has an abelian subgroup $H$ of finite index. It induces an epimorphism from $A(G)^{* *}$ on $A(H)^{* *}$, in particular $A(H)^{* *}=L^{1}(\hat{H})^{* *}$ is amenable. It follows by [G-L-W], that $\hat{H}$ is finite, and so $G$ is finite.)

If $A$ is commutative or if it possesses a continuous involution then as it is shown in [G-L], the amenability (resp. weak amenability) of $\left(A^{* *}, \circ\right)$ is equivalent to that of $\left(A^{* *}, \diamond\right)$. It seems still not known if there exists a Banach algebra $A$ for which the amenability of $\left(A^{* *}, \circ\right)$ is not equivalent to that of $\left(A^{* *}, \diamond\right)$.

The main theme of this paper is to investigate the regularity and amenabil- 
ity of the second dual of the weighted group algebras $L^{1}(G, w)$ and $M(G, w)$.

\section{Preliminaries}

Throughout this paper, $G$ is a locally compact (topological) group, and $w$ is a weight on $G$; ( which is a continuous function $w: G \rightarrow(0, \infty)$ with $w(x y) \leq w(x) w(y)$, for all $x, y \in G)$, for convenience we shall assume that $w(e)=1$, where $e$ is the identity of $G$. We define $\Omega: G \times G \rightarrow(0,1]$ by $\Omega(x, y)=w(x y) / w(x) w(y)$.

A function $h: X \times Y \rightarrow \mathbf{C}$ is said to be 0 -cluster if $\lim _{n} \lim _{m} h\left(x_{n}, y_{m}\right)=$ $0=\lim _{m} \lim _{m} h\left(x_{n}, y_{m}\right)$ for every two sequences $\left\{x_{n}\right\} \subseteq X$ and $\left\{y_{m}\right\} \subseteq Y$ of distinct points, provided the involved limits exist.

We define $w^{*}$ on $G$ by $w^{*}(x)=w(x) w\left(x^{-1}\right),(x \in G)$. It can be simply verified that $w^{*}$ is also a weight on $G$; moreover $w^{*}$ is bounded on $G$ if and only if $w$ is semi-multiplicative (that is, there exists $c>0$ such that $c w(x) w(y) \leq w(x y)$, for all $x, y \in G)$. Therefore, $\Omega$ can not be 0 -cluster when $w^{*}$ is bounded.

Define $L^{1}(G, w), L^{\infty}(G, w), G_{0}(G, w)$ and $L U C(G, w)$ as follows:

$$
\begin{aligned}
L^{1}(G, w) & =\left\{f: f w \in L^{1}(G)\right\}, \\
L^{\infty}(G, w) & =\left\{f: f / w \in L^{\infty}(G)\right\}, \\
C_{0}(G, w) & =\left\{f: f / w \in C_{0}(G)\right\}, \text { and } \\
L U C(G, w) & =\{f: f / w \in L U C(G)\} .
\end{aligned}
$$

We norm these spaces in such a way the multiplication or division by $w$ becomes an isometry between the non-weighted and the corresponding weighted spaces (whose norm will denote by $\|\cdot\|_{w}$ ). Thus the nonweighted and the corresponding weighted spaces are isometrically isomorphic as Banach spaces, but quite different as Banach algebras. Recall the inclusion relations of non-weighted cases of these spaces and the fact that $L^{1}(G)^{*}=L^{\infty}(G)$, we have:

$$
C_{0}(G, w) \subseteq L U C(G, w) \subseteq L^{\infty}(G, w)=L^{1}(G, w)^{*} .
$$

We refer the reader to [R2], for more study of different subalgebras of $L^{\infty}(G, w)$, and their equalities.

We define $M(G, w)$ such that $M(G, w)$ becomes isometric isomorphic to the Banach space $C_{0}(G, w)^{*}$. For this sake, let $M^{+}(G, w)$ be the set of 
all positive regular measures on $G$ for which $\mu w$ is again a positive regular measure on $G$; where $d(\mu w)=w d \mu$. Define an equivalence relation on $M^{+}(G, w) \times M^{+}(G, w)$ by $\left(\mu_{1}, \nu_{1}\right) \sim\left(\mu_{2}, \nu_{2}\right)$ if and only if $\mu_{1}+\nu_{2}=\mu_{2}+\nu_{1}$. Now define $M(G, w)$ by

$$
M(G, w)=\left\{[\mu, \nu]: \mu, \nu \in M^{+}(G, w)\right\},
$$

where $[\mu, \nu]$ is the equivalence class of $(\mu, \nu)$. For a full discussion on $M(G, w)$ from this point of view and the fact that $C_{0}(G, w)^{*}=M(G, w)$ see $[\mathrm{R} 1]$ and also $[\mathrm{B}]$.

It should be remarked that, if $w$ is multiplicative (i.e. $w(x y)=w(x) w(y)$, for all $x, y \in G$, or equivalently, $w$ is a positive character on $G$ ) then $L^{1}(G, w) \cong L^{1}(G)$ and $M(G, w) \cong M(G)$ as Banach algebras. Indeed it can be readily verified that $f \rightarrow f w$ and $[\mu, \nu] \rightarrow \mu w-\nu w$ are algebra isomorphism from $L^{1}(G, w)$ on $L^{1}(G)$ and $M(G, w)$ on $M(G)$, respectively.

As a ground reference for the second dual of weighted group algebras, one may refer to $[\mathrm{D}-\mathrm{L}]$.

\section{Main Results}

We start with the next lemma.

Lemma 1. If $G$ is infinite (discrete) and $\Omega$ is 0 -cluster, then $F \circ G=$ $0=F \diamond G$, for every $F, G \in l^{1}(G, w)^{* *} \backslash l^{1}(G, w)$.

Proof. Since $\Omega$ is 0 -cluster, the mapping $(x, y) \rightarrow\left(\frac{\phi}{w}\right)(x y) \Omega(x, y)$ is 0 -cluster for every $\phi \in L^{\infty}(G, w)$. Using the Example 2 in page 312 of [Y2], the mapping $(f, g) \rightarrow \phi(f \star g)=\sum \sum\left(\frac{\phi}{w}\right)(x y)(f w)(x)(g w)(y) \Omega(x, y)$ is 0 -cluster on $l^{1}(G, w) \times l^{1}(G, w)$ (the sums are taken on $\left.x, y \in G\right)$. Now for $F, G \in l^{1}(G, w)^{* *} \backslash l^{1}(G, w)$ there exist two nets $\left\{f_{\alpha}\right\}$ and $\left\{g_{\beta}\right\}$, each consisting of distinct points in $l^{1}(G, w)$ such that $f_{\alpha} \longrightarrow F$ and $g_{\alpha} \longrightarrow G$, in the weak* topology, with

$$
<F \circ G, \phi>=\lim _{\alpha} \lim _{\beta} \phi\left(f_{\alpha} \star g_{\beta}\right) \text { and }<F \diamond G, \phi>=\lim _{\beta} \lim _{\alpha} \phi\left(f_{\alpha} \star\right.
$$
$\left.g_{\beta}\right)$,

for every $\phi \in L^{\infty}(G, w)$. One can construct two subsequences $\left\{f_{\alpha_{m}}\right\}$ and $\left\{g_{\beta_{n}}\right\}$ of $\left\{f_{\alpha}\right\}$ and $\left\{g_{\beta}\right\}$, respectively, such that, $\langle F \circ G, \phi>=$ $\lim _{m} \lim _{n} \phi\left(f_{\alpha_{m}} \star g_{\beta_{n}}\right)=0=\lim _{n} \lim _{m} \phi\left(f_{\alpha_{m}} \star g_{\beta_{n}}\right)=<F \diamond G, \phi>$, as required.

Now, we come to the one of the main results. 
Theorem 2. The following statements are equivalent.

(i) $L^{1}(G, w)$ is regular,

(ii) $G$ is finite or $G$ is discrete and $\Omega$ is 0 -cluster,

(iii) $L^{1}(G, w)^{* *}$ is regular.

Proof. For (i) $\Rightarrow($ ii $)$, suppose that $L^{1}(G, w)$ be regular. Since $L^{1}(G, w)$ is weakly sequentially complete and admits a bounded approximate identity, it is unital by theorem 3.3 of [U1]. Therefore $G$ is discrete. If $G$ is infinite, then by corollary 3.8 of [B-R] $\Omega$ must be 0 -cluster. For (ii) $\Rightarrow($ iii), if $G$ is finite then $L^{1}(G, w)$ is reflexive; for the infinite case (iii) follows from Lemma1.

Suppose that $G$ admits a multiplicative weight bounded by $w$, (for instance, it is the case if either $1 \leq w$ or $G$ is amenable (as a group), for the latter see Lemma 1 of $[\mathrm{W}]$ ). Then, there exists a unique multiplicative weight on $G$ which is equivalent to $w$, provided $w^{*}$ is bounded. Indeed, $\varphi(x)=\lim _{n \rightarrow \infty} w\left(x^{n}\right)^{1 / n}$ defines a multiplicative weight on $G$ with $\varphi \leq w \leq c \varphi$, in which $c=\sup _{x \in G} w^{*}(x)$; see [W] for further details. In particular, $L^{1}(G, w)=L^{1}(G, \varphi) \cong L^{1}(G)$ and $M(G, w)=M(G, \varphi) \cong M(G)$.

An elegant result of [Gro] states $L^{1}(G, w)$ is amenable if and only $G$ is amenable and $w^{*}$ is bounded. Therefore, $L^{1}(G, w)$ is amenable if and only if $G$ is amenable and $L^{1}(G, w) \cong L^{1}(G)$. Recently, it has been proved in [D-G-H] that $M(G)$ is amenable if and only if $G$ is amenable and discrete. As a weighted version of this we have;

Proposition 3. $M(G, w)$ is amenable if and only if $G$ is amenable, discrete and $w^{*}$ is bounded.

Proof. If $M(G, w)$ is amenable, then $L^{1}(G, w)$ is amenable, therefore $G$ is amenable and $w^{*}$ is bounded; and so by the discussion just before the proposition, there exists a unique multiplicative weight on $G$ equivalent to $w$. It implies that, $M(G, w) \cong M(G)$. In particular, $M(G)$ is amenable. By [D-G-H] $G$ must be discrete. Since in the discrete setting $M(G, w)=$ $L^{1}(G, w)$, the converse follows from [Gro]

As the second main result we have the next which is an extension of Theorem 1.3 of [G-L-W].

Theorem 4. The following statements are equivalent.

(i) $L^{1}(G, w)^{* *}$ is amenable,

(ii) $L^{1}(G, w)$ is amenable and regular, 
(iii) $L^{1}(G, w)$ is regular and $w^{*}$ is bounded,

(iv) $L^{1}(G, w)$ is reflexive and $w^{*}$ is bounded,

(v) $L^{1}(G, w)$ is a $C^{*}$-algebra,

(vi) $G$ is finite.

Proof. Trivially (vi) implies the other parts. If $L^{1}(G, w)^{* *}$ is amenable, then so is $L^{1}(G, w)$, and so $L^{1}(G, w) \cong L^{1}(G)$. Now the amenability of $L^{1}(G)^{* *}$ necessitates $G$ must be finite by Theorem 1.3 of [G-L-W]. Thus (i) $\Rightarrow$ (vi) follows. The implications (ii) $\Rightarrow$ (iii) and (iv) $\Rightarrow$ (iii) are obvious. Let $L^{1}(G, w)$ be regular and $w^{*}$ be bounded; therefore $\Omega$ can not be 0 -cluster and by Theorem 2, $G$ is finite. Assume that $L^{1}(G, w)$ is a $C^{*}$-algebra; then it is regular and so $G$ is discrete. Moreover, the equality $\left\|\delta_{x} * \delta_{x}^{*}\right\|_{w}=\left\|\delta_{x}\right\|_{w}^{2}$, for every $x \in G$ implies that $w(x)=\Delta(x)^{1 / 2}$, for each $x \in G$ ( $\Delta$ is the modular function of $G$ ), and this implies that $w$ is multiplicative and so $\Omega=1$. Now Theorem 2 implies that $G$ is finite and this completes the proof.

Remarks. (i) The conclusions of Theorems 2 and 4 remains valid if we replace $L^{1}(G, w)$ by $M(G, w)$.

(ii) For a Banach algebra $A$ if $A^{* * *} \cdot F=A^{*} \diamond F$, for every $F \in A^{* *}$, ( where $<m \cdot F, G>=<m, F \circ G>$ for every $m \in A^{* * *}, F, G \in A^{* *}$ ) then it is not hard to prove that the regularity of $A^{* *}$ is equivalent to that of $A$; (indeed if $A$ is regular, then for every $f \in A^{*}$, the mapping $F \rightarrow f \diamond F: A^{* *} \rightarrow A^{*}$ is weakly compact, and the equality $A^{* * *} \cdot F=A^{*} \diamond F$ implies that for every $\Phi \in A^{* * *}$ the mapping $F \rightarrow \Phi \cdot F: A^{* *} \rightarrow A^{* * *}$ is weakly compact, which is equivalent to the regularity of $\left.A^{* *}\right)$. Using this fact, one may give a different proof to the Theorem 2 .

(iii) For a Banach algebra $A$ with a bounded approximate identity of norm one, $A^{*} A$ is a closed subspace of $A^{*}$, and $A^{* *}=\left(A^{*} A\right)^{*} \oplus\left(A^{*} A\right)^{\perp}$ (as Banach spaces), where $\left(A^{*} A\right)^{\perp}=\left\{F \in A^{* *}: A^{* *} \circ F=0\right\}$ is a closed ideal of $\left(A^{* *}, \circ\right)$ and $\left(A^{*} A\right)^{*}$ is a closed subalgebra of $\left(A^{* *}, \circ\right)$. These observations together with the Lemma 2.3 of [L-L] imply that; if $\left(A^{* *}, \circ\right)$ is weakly amenable then so is $\left(A^{*} A\right)^{*}$. Now for $A=L^{1}(G, w)$ it has been shown in Proposition 1.3 of [Gro] that $A^{*} A=\operatorname{LUC}(G, w)$. On the other hand, using the methods of Lemma 1.1 of [G-L-L], we have $\operatorname{LUC}(G, w)^{*}=M(G, w) \oplus$ $C_{0}(G, w)^{\perp}$, and that $M(G, w), C_{0}(G, w)^{\perp}$ are closed subalgebra and closed ideal of $\operatorname{LUC}(G, w)^{*}$, respectively. Again use Lemma 2.3 of [L-L] the weak amenability of $L^{1}(G, w)^{* *}$ implies that of $M(G, w)$, which is an extension of Proposition 4.14 in [L-L]. 
(iv) The existing examples support the conjecture that, for a Banach algebra $A$ if $A^{* *}$ is amenable then $A$ is regular.

\section{References}

[A] Arens R.I.," The adjoint of a bilinear operation ", Proc. Amer. Math. Soc. 2, pp. 839-848, (1951).

[B] Baker J.W.," Measure algebras on semigroups", The analytical and topological theory of semigroups, Walter de Gruyter, Berlin, New York, pp. 221-252, (1990).

[B-R] Baker J.W. and Rejali A., " On the Arens regularity of the weighted convolution algebras, J. London Math. Soc. (2) 40, pp. 535-546, (1987).

[D-G-H] Dales H.G., Ghahramani F. and Helemskii A.Y.A., " The amenability of measure algebras", J. London Math. Soc. (2) 66, pp. 213-226, (2002).

[D-H] Duncan J. and Houssainun S.A.R., " The second dual of a Banach algebra", Proc. Roy. Soc. Edinburgh A 84, pp. 309-325, (1979).

[D-L] Dales H.G. and Lau A.T.-M.," The second duals of Beurling algebras", Mem. Amer. Math. Soc. 177, No. 386, (2005).

[F-R] Forrest B. and Runde V.," Amenability and weak amenability of the Fourier algebra", Math. Z. 250 4, pp. 731-744, (2005).

[G-L] Ghahramani F. and Laali J." Amenability and topological centers of the second duals of Banach algebras", Bull. Austral. Math. Soc. 65, pp. 191-197, (2002).

[G-L-L] Ghahramani F., Lau A.T.M. and Losert V.," Isometric isomorphisms between Banach algebras related to locally compact groups", Trans, Amer. Math. Soc. 321, pp. 273-383, (1990).

[G-L-W] Ghahramani F., Loy R.J. and Willis G.," Amenability and weak amenability of second conjugate Banach algebras", Proc. Amer. Math. Soc. 124, pp. 1489-1497, (1996).

[Go] Gourdeau F., "Amenability and the second dual of a Banach algebra", Studia Math. 125, pp. 45-80, (1997). 
[Gro] Gronback N.," Amenability of weighted convolution algebras on locally compact groups", Trans. Amer. Math. Soc. 319, pp. 765-775, (1990).

[Gra] Granirer E.," Amenability and semisimplicity for second duals of quotients of the Fourier algebra $A(G)$, , J. Austral. Math. Soc. (Series A) 63, pp. 289-296, (1997).

[L-L] Lau A.T.M and Loy R.J., " Weak amenability of Banach algebras on locally compact groups", J. Funct. Anal. 145, pp. 175-204, (1997).

[P] Pym J.S., "Remarks on the second duals of Banach algebras", Nigerian Math. Soc. 2, pp. 31-33, (1983).

[R1] Rejali A., " The analogue of weighted group algebra for semitopological semigroups", J. Sci. I.R. Iran 6, pp. 113-120, (1995).

[R2] Rejali A.," Weighted function spaces on topological groups", Bull. Iranian Math. Soc. 22, pp. 43-63, (1996).

[S] Sherman S., " The second adjoint of a $C^{*}$-algebra," Proc. Intern. Congr. Math. Cambridge, I, 470, (1950).

[U1] Ulger A.," Arens regularity of weakly sequentially complete Banach algebras", Proc. Amer. Math. Soc. 127, pp. 3221-3227, (1999).

[U2] Ulger A.," Centeral elements of $A^{* *}$ for certain Banach algebras $A$ without bounded approximate identities," Glasgow Math. J. 41, pp. 369-377, (1999).

[W] White M., "Characters on weighted amenable groups", Bull. London Math. Soc. 23, pp. 375-380, (1991).

[Y1] Young N.," The irregularity of multiplication in group algebras," Quart. J. Math. Oxford (2) 24, pp. 59-62, (1973).

[Y2] Young N.," Separate continuity and multilinear operations", Proc. London Math. Soc. (3) 26, pp. 289-319, (1973).

[Y3] Young N.," Periodicity of functionals and representations of normed algebras on reflexive spaces," Proc. Edinburgh Math. Soc. 20, pp. 100-120, (1976). 
A. Rejali

Department of Mathematics

Isfahan University

Isfahan 81746-73441

Iran

e-mail : rejali@sci.ui.ac.ir

and

H. R. E. Vishki

Faculty of Mathematical Sciences

Ferdowsi University, Mashhad

P.O. Box 91775-1159

Iran

e-mail : vishki@ferdowsi.um.ac.ir 